\title{
Time Series Similarity Method Research of UAVs Combat Situation Advantage Information
}

\author{
Xia Chen ${ }^{1}$, Yifei Wang ${ }^{2}$ and Tuo Wang ${ }^{3}$ \\ ${ }^{1,2}$ Shenyang Aerospace University, Automation Department, Shenyang, China \\ ${ }^{3}$ Shenyang Aerospace University, Economics and Management Department \\ 330967185@qq.com,xiachen1108@163.com,804187441@qq.com
}

\begin{abstract}
In order to solve UAVs countermeasure selection problem under the complex air combat situation, we proposed to use Time Series Similarity Method to quantitative compare the combat situation information of adjacent time and divide the game situation into "change" or "stable" situation. Firstly, the paper analyzed the variability of real-time combat situation, collected the enemy situation information over a period of time, used the advantage of the situation function to calculate the advantage of our situation and list the advantages of the overall trend information sequence. Then we proposed a Time Series Similarity method of UAVs combat situation advantage, made the curve of the situation advantage and determined the stability good or not. Made the assessment of the game battlefield environment situation accurately and then selected the most realistic combat countermeasure method that conducive to battlefield environment. At last, we took an example, two sides $1 \mathrm{~V} 1$ air combat, using the method proposed in this paper for checking. The result showed that the method feasibility and effectiveness.
\end{abstract}

Key Words: air combat; situation advantage; Time Series; Similarity function

\section{Introduction}

With the advancement of modern aviation technology and the needs of military combat, multi-UAV air combat is the main trends in the future. It is one of the key technologies for UAV to make an accurate and timely decision in a complex combat environment [1]. Currently, the air combat countermeasures research has attracted the attention of scholars and experts at home and abroad. The main technology: Differential Game [2-4], Intelligent Differential Game [5], Expert system [6], Neural Network [7] and Game Theory Law [8], etc. It is noted that, although some experts and scholars in the UAV tactical countermeasures technology has made a lot of research results, However, each countermeasures method has its own advantages and disadvantages. Differential Game and Intelligent Differential Game are the countermeasures method considered the continuous time that can reflect the real-time combat situation better, but this method is more difficult for modeling and solving. Expert system is a problem-solving method based on experience and knowledge, but its form has been fixed and it is hard to fit for complex combat environments characteristics. Neural network can simulate the human brain with strong data processing capabilities and fault tolerance ability, but it requires a large number of training samples, and usually it is difficult to obtain a large number of training samples. Game Theory is a rational behavior strategy that studies interest mutual restraint of both sides, however, this method is considered discrete time countermeasures law, so it is difficult to reflect the real-time combat situation and dynamics. Therefore, in actual combat countermeasures, how to make a strategy choice to solve problems in air combat situation based on complex environments is an important research question in UAV combat techniques that needs to be addressed.

Time Series are arranged in chronological order of the various observations recorded 
data sets. Time Series are widespread in various fields, such as stock prices changes in financial and securities markets every day; Daily sales of a commodity in commercial retail industry; Weather forecasts, a regional daily temperature and pressure readings; and biomedical among the symptoms of a patient's heart rate variation at each time and so on. In recent years, Time Series Similarity has become a hot research topic. Paper[9] Based on the k-means clustering algorithm, Linear time series segmentation based on overall sequence similarity segmentation model, considered the upper and lower bounds of sequence features, proposed a k-edge clustering algorithm based on Time Series Similarity. Paper [10] used of the symbol sequence characteristics of Time Series, proposed a acquaintance of measurement methods, the symbol of the statistical vector space method. Paper [11] proposed a partial segment of dynamic time warping algorithm, improved the classical dynamic time warping algorithm efficiency effectively. From the published literature, study Time Sequence similarity problem has achieved preliminary research results, there are still many important questions remain unresolved. One important issue is the Time Sequence Similarity of the combat situation. Due to the complexity and transient of the battlefield environment, leading to the air combat situation information between two sides changes over time. Different situation information may result in different decisions. Therefore, the study of Time Series Similarity in combat situation superiority based on dynamic information is an important issue in air combat decision. So far, there is no reported in the literature for air combat situation advantage of the Time Series Similarity problems. UAV combat situation superiority of the Time Series Similarity problems under the complex environment has not been resolved. This article is based on the two sides of the real-time dynamics of sensory information, extract Time Series data of different time, build the situation advantage collection of Time Series and give a reasonable Time Sequence Similarity method for the UAV aerial superiority situation. The method quantifies the Time Series Similarity of the situation advantages, makes the assessment of the game battlefield environment situation accurately, divides the game situation into "change" or "stable" situation, thus provides a scientific basis for choosing the most beneficial method of combat countermeasures for the actual battlefield environment.

\section{UAV Situational Advantage Definitions}

\subsection{The Advantage Function of Angle}

According to literature, give the related definition of both sides of the UAV.

In Figure $1, \mathrm{~F}$ is our UAV and $\mathrm{T}$ is the enemy, $\mathrm{R}$ is the distance between $\mathrm{F}$ and $\mathrm{T}$. $v_{F}$ is the speed of our UAV and $v_{T}$ is the speed of the enemy's which is measured by our UAV. FT is the line of sight that connected $\mathrm{F}$ and $\mathrm{T}$. azimuth of target $\mathrm{:}$ : the angle between F's heading and the line FT, in order to track the target, we should keep . Course angle ? of target: the angle between line FT and T's heading.

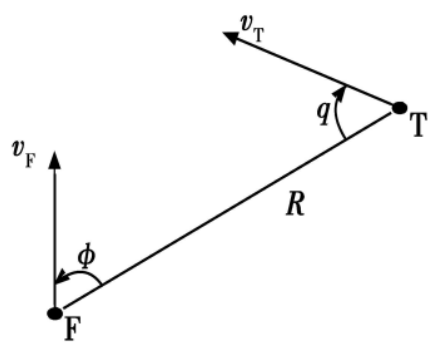

Figure 1. 1v1 Air Combat Situation Schematic Diagram 
As the definition above, according to literature, we have the advantage function of the angle [12]:

$T_{a}=\frac{|q|-|\phi|}{180^{\circ}}$

\subsection{The Advantage Function of Speed}

The higher speed of combat aircraft, the stronger the attack advantage. According to the literature [13], given the speed advantage function:

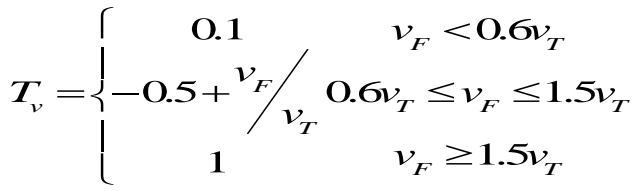

In the formula, $v_{F}$ is the speed of the attack aircraft; $v_{T}$ was the target speed.

\subsection{The Advantage Function of Distance}

According to the literature [14-15], the missile carried by UAV has a minimum firing distance $R_{\text {min }}$, and the maximum firing distance is $R_{\max }$, we have the distance advantage function similar to Gaussian distribution:

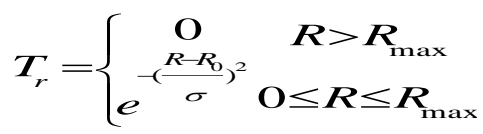

In the formula,

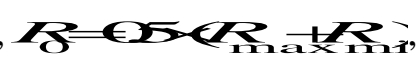

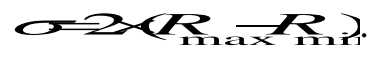

\subsection{Combat Situation Advantages Set}

When we analysis a comprehensive situation of the actual combat, consider the variability of real-time combat situation, as well as the different situation advantage has a different influence degree for the combat decision-making. We weighted the angle advantage, the speed advantage, and the distance advantage. So the whole situation advantage at $\mathrm{t}$ time expressed as:

$$
S_{t}=\left\{w_{1} \cdot T_{t}^{a}, w_{2} \cdot T_{t}^{v}, w_{3} \cdot T_{t}^{d}\right\}
$$

In the formula $w_{1}, w_{2}, w_{3}$ are the weighted coefficients, $0 \leq w_{1}, w_{2}, w_{3} \leq 1$ 


\subsection{Combat Situation Advantages Set of Time Series Similarity}

$S_{t}$ is the situation advantage set at time t, Time Series is $\left\{t_{1}, \cdots, t_{k}, \cdots, t_{n}\right\}$, so the situation advantage set expressed as:

$S=\left\{S_{t_{1}}, \cdots, S_{t_{k}}, \cdots, S_{t_{n}}\right\}$

In the formula, $S_{t_{1}}$ is the situation advantage set at $t_{1}$ time, $S_{t_{k}}$ is the situation advantage set at $t_{k}$ time, $S_{t_{n}}$ is the situation advantage set at $t_{n}$ time. So the situation advantage set can also be expressed as follows:

$S=\left\{\left\{w_{1} \cdot T_{t_{1}}^{a}, w_{2} \cdot T_{t_{1}}{ }^{v}, w_{3} \cdot T_{t_{1}}^{d}\right\}, \cdots,\left\{w_{1} \cdot T_{t_{2}}^{a}, w_{2} \cdot T_{t_{2}}{ }^{v}, w_{3} \cdot T_{t_{2}}^{d}\right\}, \cdots,\left\{w_{1} \cdot T_{t_{n}}^{a}, w_{2} \cdot T_{t_{n}}^{v}, w_{3} \cdot T_{t_{n}}^{d}\right\}\right\}$

\section{Time Series Similarity Method of UAVs Combat Situation Advantage}

In actual combat environment, due to the complexity and transient of the battlefield environment, the air combat situation information between two sides changes over time. The dynamic real-time situational information directly affect combat advantage of the situation, different advantage of the situation lead to a different decision results. "Stable situation" refers to the trend advantages of UAV changes minor in any two adjacent times. "Change situation" refers to the trend advantages of UAV changes large in any two adjacent times [16]. This paper, based on real-time situational information, proposed Time series similarity method of the situation advantages of UAVs combat.

\subsection{Time Series Similarity Method of UAVs Combat Situation Advantage}

This article discrete time $t$, Time series $\left\{t_{1}, \cdots, t_{k}, \cdots, t_{n}\right\}$, we can get the combat situation of the Time Series, the situation advantage set at $t_{k-1}$ is $\left\{w_{1} \cdot T_{t_{k-1}}^{a}, w_{2} \cdot T_{t_{k-1}}^{v}, w_{3} \cdot T_{t_{k-1}}^{d}\right\}$, the situation advantage set at $t_{k}$ is $\left\{w_{1} \cdot T_{t_{k}}^{a} w_{2} \cdot T_{t_{k}}{ }^{v}, w_{3} \cdot T_{t_{k}}{ }^{d}\right\}$, so the difference value of situation advantage changes between $t_{k-1}$ and $t_{k}$ is:

$$
\Delta_{k}=\sqrt{\left(w_{1} \cdot T_{t_{k}}^{a}-w_{1} \cdot T_{t_{k-1}}^{a}\right)^{2}+\left(w_{2} \cdot T_{t_{k}}^{v}-w_{2} \cdot T_{t_{k-1}}^{v}\right)^{2}+\left(w_{3} \cdot T_{t_{k}}^{d}-w_{3} \cdot T_{t_{k-1}}^{d}\right)^{2}}
$$

From formula (7), we can see when the situation advantage at $t_{k-1}$ and $t_{k}$ are the same, the difference value $\Delta_{k}=0$, the situation advantage at $t_{k-1}$ and $t_{k}$ more different, the difference value $\Delta_{k}$ larger.

Set the situation advantage threshold of the adjacent time $\rho_{0}$, the situation advantage similarity function is:

$$
\rho\left(t_{k-1}, t_{k}\right)=1-\frac{\Delta_{k}}{\sqrt{\left(w_{1} \cdot T_{t_{k-1}}^{a}\right)^{2}+\left(w_{2} \cdot T_{t_{k-1}}^{v}\right)^{2}+\left(w_{3} \cdot T_{t_{k-1}}^{d}\right)^{2}}+\sqrt{\left(w_{1} \cdot T_{t_{k}}^{a}\right)^{2}+\left(w_{2} \cdot T_{t_{k}}^{v}\right)^{2}+\left(w_{3} \cdot T_{t_{k}}^{d}\right)^{2}}}
$$

If $\rho\left(t_{k-1}, t_{k}\right) \geq \rho_{0}$, we say the situation advantage between $t_{k-1}$ and $t_{k}$ are stable, otherwise we say the situation advantage is unstable.

From formula (8), $\rho\left(t_{k-1}, t_{k}\right) \leq 1$, The larger the value, the better the similarity, when the situation advantage at $t_{k-1}$ and $t_{k}$ are the same, the similarity function $\rho\left(t_{k-1}, t_{k}\right)=1$, when the situation advantage at $t_{k-1}$ and $t_{k}$ are quite different, the similarity function $\rho\left(t_{k-1}, t_{k}\right)=1$. So in the UAVs air combat game, according to the situation 
advantage similarity function, we can divide the situation advantage into "Stable situation" and "Change situation". If we sample n points in a period of time, compare the situation advantage function between every two adjacent time, then we can get n-1 similarity function value $\rho\left(t_{k-1}, t_{k}\right)$, compare $\rho\left(t_{k-1}, t_{k}\right)$ with $\rho_{0}$, if $\rho\left(t_{k-1}, t_{k}\right) \geq \rho_{0}$, we say the situation advantage of $t_{k-1}$ and $t_{k}$ is stable, otherwise the situation advantage is unstable. If all of these $\mathrm{n}-1$ function values meet $\rho\left(t_{k-1}, t_{k}\right) \geq \rho_{0} k=1,2, \cdots, n$, we say that our UAVs air combat situation advantage is stable between $t_{1}$ and $t_{n}$. We can use Game Theory method and find the optimal decision strategy. If one or more of these n-1 function values do not meet $\rho\left(t_{k-1}, t_{k}\right) \geq \rho_{0} k=1,2, \cdots, n$, we say that our UAVs air combat situation advantage is unstable between $t_{1}$ and $t_{n}$, we can use Differential Game method, establish real-time combat mathematical model, use constructed Hamilton function to find the optimal solution of the model and give the best differential games.

\subsection{Method Steps}

Step 1 Sampling interval enemy's situation information over time;

Step 2 from formula (1)-(3), we calculate the advantage of angle,

the advantage of speed and the advantage of distance;

Step 3 according to formula (4), calculate the situation advantage set at t;

Step 4 according to formula(5)and(6), calculate the time series situation advantage set between $t_{1}$ and $t_{n}$.

Step 5 according to formula(7)and(8), calculate the situation advantage similarity function value $\rho\left(t_{k-1}, t_{k}\right)$ between the Adjacent time $t_{k-1}$ and $t_{k} k=1,2, \cdots, n$.

Step 6 compare the value $\rho\left(t_{k-1}, t_{k}\right)$ with the threshold $\rho_{0}$, and judge if the situation advantage similarity between $t_{k-1}$ and $t_{k}$ is stable or not;

Step 7 If the $\mathrm{n}-1$ similarity functions of $\mathrm{n}$ sample points satisfy the stability condition $\rho\left(t_{k-1}, t_{k}\right) \geq \rho_{0} \quad(k=1,2, \cdots, n)$, then the advantages of our air combat situation is relatively stable. If one or more function value of the n- 1 similarity function do not satisfied stability condition, the advantages of our air combat situation changed greatly.

\section{Examples Analysis}

UAV 1V1 game situation analysis, we intercept enemy UAV drones, two time points sampled. In the period, we sampled the enemy UAV situational information, between 1 and $t+\sigma$, sampled once every $5 \mathrm{~s}$, a total of 10 samples, the following parameters:

Table 1. The Parameters of Speed, Angle and Distance of Two Sides for

\begin{tabular}{cccccccc}
\multicolumn{7}{c}{$t_{1} t_{10}$} \\
\hline \hline Sampling time & $v_{a}(\mathrm{~m} / \mathrm{s})$ & $v_{b}(\mathrm{~m} / \mathrm{s})$ & $\phi \quad\left(^{\circ}\right)$ & $q\left(^{\circ}\right)$ & $R \quad(k m)$ \\
\hline$t_{1}$ & 75 & 60 & 30 & 42 & 38 \\
$t_{2}$ & 78 & 62 & 32 & 40 & 36 \\
$t_{3}$ & 80 & 65 & 35 & 39 & 35 \\
$t_{4}$ & 85 & 65 & 36 & 40 & 33 \\
$t_{5}$ & 100 & 80 & 34 & 55 & 30 \\
$t_{6}$ & 120 & 80 & 30 & 68 & 28 \\
\hline \hline
\end{tabular}




\begin{tabular}{cccccc}
\hline \hline$t_{7}$ & 120 & 78 & 28 & 60 & 25 \\
$t_{8}$ & 112 & 100 & 25 & 35 & 23 \\
$t_{9}$ & 110 & 102 & 30 & 30 & 20 \\
$t_{10}$ & 115 & 105 & 26 & 45 & 18 \\
\hline \hline
\end{tabular}

between $_{t^{\prime}}$ and $t_{t^{\prime}+\sigma}$, sampled once every $5 \mathrm{~s}$, a total of 10 samples, the following parameters:

Table 2. The Parameters of Speed, Angle and Distance of Two Sides for

$$
t_{1}^{\prime}-t_{10}^{\prime}
$$

\begin{tabular}{cccccc}
\hline \hline Sampling time & $v_{a}(\mathrm{~m} / \mathrm{s})$ & $v_{b}(\mathrm{~m} / \mathrm{s})$ & $\phi\left(^{\circ}\right)$ & $q\left(^{\circ}\right)$ & $R(\mathrm{~km})$ \\
\hline$t_{1}^{\prime}$ & 108 & 100 & 33 & 35 & 32 \\
$t_{12}^{\prime}$ & 110 & 98 & 32 & 33 & 28 \\
$t_{3}^{\prime}$ & 112 & 104 & 30 & 39 & 27 \\
$t_{4}^{\prime}$ & 109 & 100 & 35 & 40 & 26 \\
$t_{5}^{\prime}$ & 110 & 105 & 38 & 45 & 25 \\
$t_{6}^{\prime}$ & 113 & 105 & 36 & 46 & 24 \\
$t_{7}^{\prime}$ & 112 & 108 & 38 & 44 & 25 \\
$t_{8}^{\prime}$ & 115 & 100 & 36 & 45 & 24 \\
$t_{9}^{\prime}$ & 109 & 102 & 33 & 47 & 22 \\
$t_{10}^{\prime}$ & 116 & 105 & 35 & 48 & 20 \\
\hline \hline $\mathrm{R}_{\max }=45 \mathrm{~km}, R_{\min }=0.5 \mathrm{~km}$ & & &
\end{tabular}

According to formula (1)-(3), we can get the angle advantage, the speed advantage and the distance advantage of ours, Coefficient $k_{1}=0.4$, Coefficient $k_{2}=0.3$, Coefficient $k_{3}=0.3$, the flight path of two sides and the sampling points are as follows:

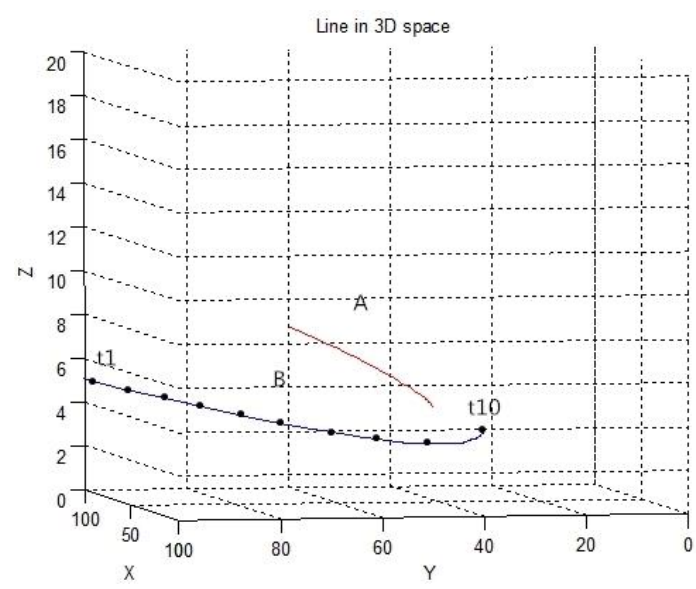

Figure 2. The Flight Path of Two Sides and the Sampling Points from $t$ to $t+\sigma$ 


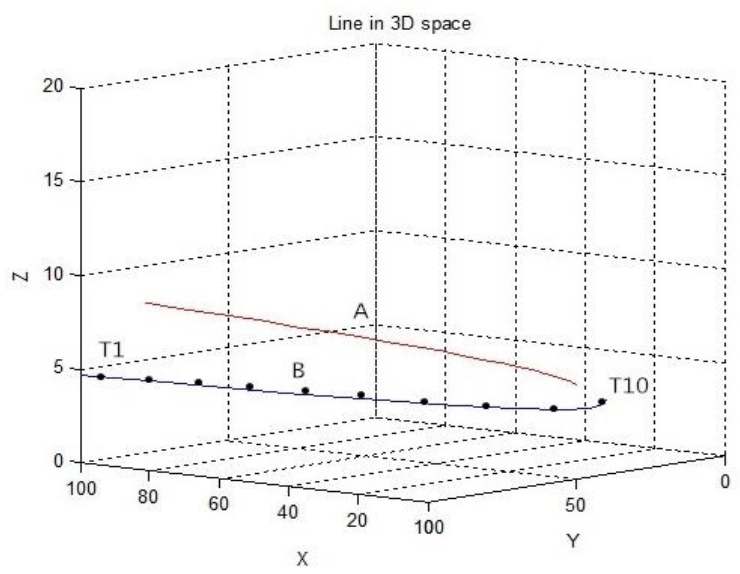

Figure 3. The Flight Path of Two Sides and the Sampling Points from

$$
t^{\prime} \mathbf{t} \mathbf{o} t^{\prime}+\sigma
$$

Between $t^{\prime}$ and $t^{t^{\prime}+\sigma}$, sampled once every $5 \mathrm{~s}$, a total of 10 samples, the following parameters:

Table 3. The Situation Advantages Set of Two Sides in Two Periods

\begin{tabular}{|c|c|c|c|}
\hline $\begin{array}{l}\text { Sampling } \\
\text { time }\end{array}$ & Situation advantage & $\begin{array}{l}\text { Sampling } \\
\text { time }\end{array}$ & S Situation advantage \\
\hline$t_{1}$ & $s_{t_{1}}=\{0.0267,0.2250,0.2913\}$ & $t_{1}^{\prime}$ & $S_{t^{\prime} 1}=[0.0044,0.1740,0.2968]$ \\
\hline$t_{2}$ & $s_{t 2}=\left[\begin{array}{lll}0.0178, & 0.2274, & 0.2934\end{array}\right]$ & $t_{2}^{\prime}$ & $S_{t^{\prime 2}}=\left[\begin{array}{lll}0.0022, & 0.1867, & 0.2990\end{array}\right]$ \\
\hline$t_{3}$ & $s_{t 3}=\left[\begin{array}{lll}0.0089, & 0.2192, & 0.2944\end{array}\right]$ & $t_{3}^{\prime}$ & $S_{i 3}=\left[\begin{array}{ll}0.0199, & 0.1731,0.2993\end{array}\right]$ \\
\hline$t_{4}$ & $s_{t 4}=\left[\begin{array}{lll}0.0089, & 0.2423, & 0.2960\end{array}\right]$ & $t_{4}^{\prime}$ & $S_{t^{\prime} 4}=[0.0111,0.1770,0.2995]$ \\
\hline$t_{5}$ & $S_{t 5}=\left[\begin{array}{lll}0.0467, & 0.2250, & 0.2980\end{array}\right]$ & $t_{5}^{\prime}$ & $S_{t_{5}}=\left[\begin{array}{ll}0.0155,0.1643,0.2998\end{array}\right]$ \\
\hline$t_{6}$ & $S_{t 6}=\left[\begin{array}{lll}0.0844, & 0.3000, & 0.2990\end{array}\right]$ & $t_{6}^{\prime}$ & $S_{t^{\prime 6}}=\left[\begin{array}{lll}0.0222, & 0.1729, & 0.2997\end{array}\right]$ \\
\hline$t_{7}$ & $s_{t 7}=\left[\begin{array}{lll}0.0711, & 0.3000, & 0.2998\end{array}\right]$ & $t_{7}^{\prime}$ & $S_{i 7}=[0.0133,0.1611,0.2998]$ \\
\hline$t_{8}$ & $s_{t 8}=\left[\begin{array}{lll}0.0222, & 0.1860, & 0.3000\end{array}\right]$ & $t_{8}^{\prime}$ & $S_{t^{\prime} 8}=\left[\begin{array}{lll}0.0199, & 0.1950,0.2997\end{array}\right]$ \\
\hline$t_{9}$ & $s_{t 9}=\left[\begin{array}{lll}0.0000, & 0.1735, & 0.2997\end{array}\right]$ & $t_{9}^{\prime}$ & $S_{t^{\prime} 9}=\left[\begin{array}{lll}0.0311, & 0.1706, & 0.2999\end{array}\right]$ \\
\hline$t_{10}$ & $s_{t 10}=\left[\begin{array}{lll}0.0422, & 0.1786, & 0.2992\end{array}\right]$ & $t_{10}^{\prime}$ & $S_{t 10}=[0.0288,0.1814,0.2997]$ \\
\hline
\end{tabular}

According to formula (4)-(6), we can get the Time series situation advantage set of $t_{1}-t_{10}$ and $t_{1}^{\prime}-t_{10}^{\prime}$ : According to formula (7) and (8), we can calculate the correlation coefficient $t_{1} \sim t_{10}$ :

$$
\begin{aligned}
& \rho_{12}=0.9875 \rho_{23}=0.9836 \rho_{34}=0.9691 \rho_{45}=0.9452 \rho_{56}=0.8961 \rho_{67}=0.9845 \rho_{78}=0.8417 \\
& \rho_{89}=0.9636 \rho_{910}=0.9390 。
\end{aligned}
$$

Similarly, we can calculate the correlation coefficient of $t_{1}^{\prime} \sim t_{10}^{\prime}$ :

$$
\begin{aligned}
& \rho_{12}=0.9812 \rho_{23}=0.9681 \rho_{34}=0.9861 \rho_{45}=0.9805 \rho_{56}=0.9842 \rho_{67}=0.9785 \quad \rho_{78}=0.9506 \\
& \rho_{89}=0.9619 \rho_{910}=0.9842 。
\end{aligned}
$$


Set the threshold $\rho_{0}=0.95$, draw the situation advantage similar trends curves of these two period as follows:

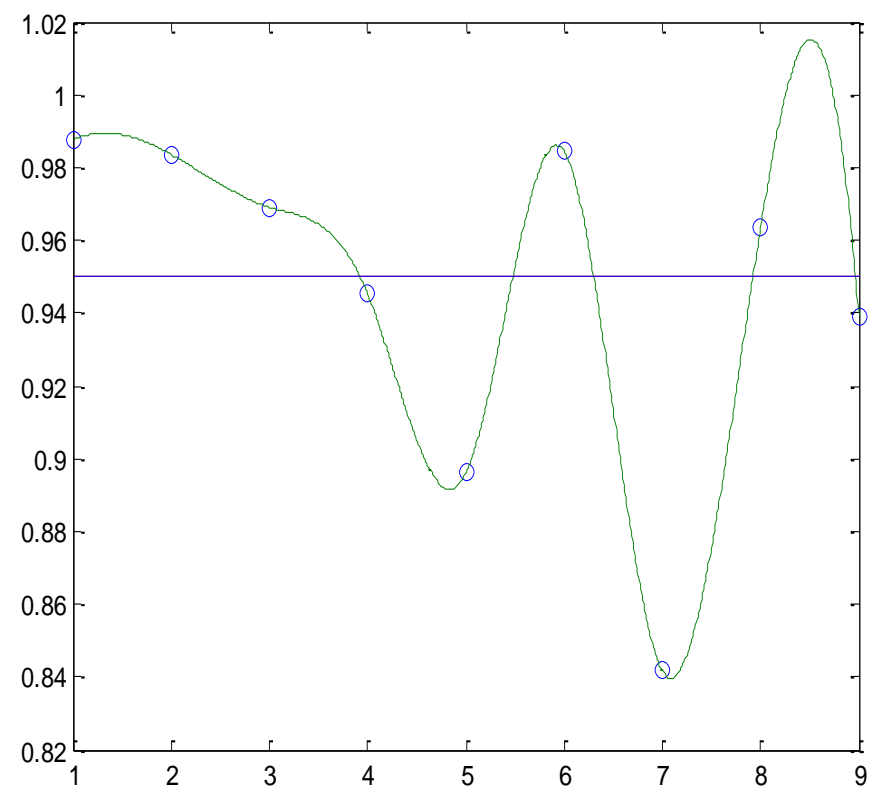

Figure 4. Situation Advantage Similarity Trend Change Figure of $t-t+\sigma$

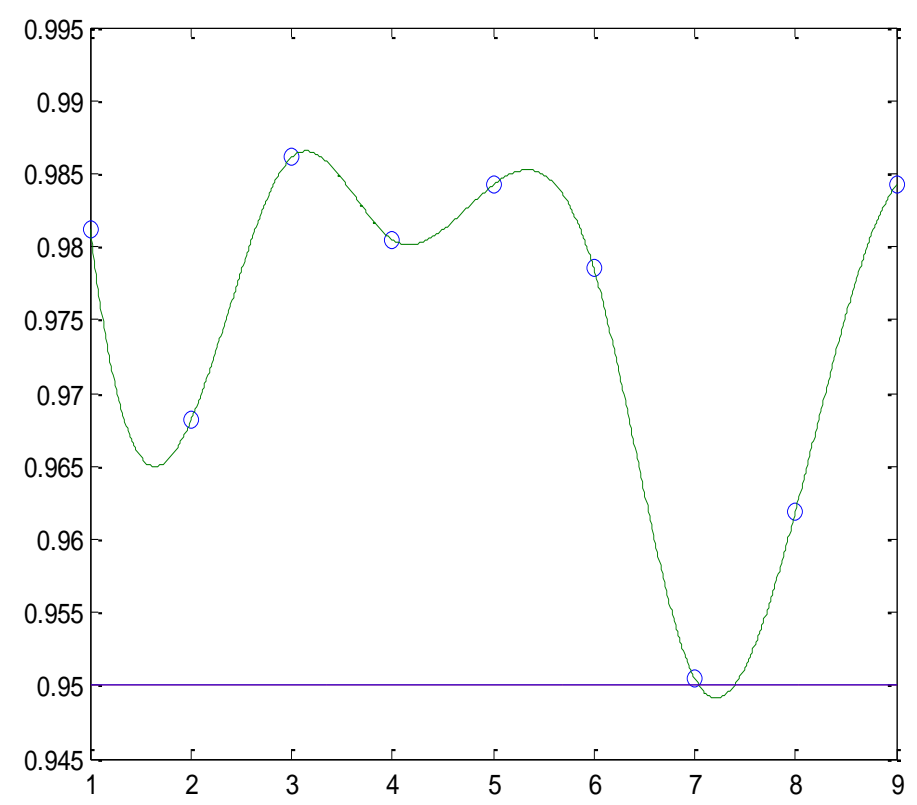

Figure 5. Situation Advantage Similarity Trend Change Figure of $t^{\prime}-t^{\prime}+\sigma$

We can see from Figure 4, the values points 4,5,7,9 are smaller than the threshold $\rho_{0}=0.95$, especially the point 5 and 7 , the values of $\rho_{56}$ and $\rho_{78}$ are small, so in the period $t_{5} \sim t_{6}$ and $t_{7} \sim t_{8}$, the situation advantage changes a lot, the game 
situation is unstable, we choose the Differencial Game method and give the best air combat strategy.

We can see from Figure 5, all of the correlation coefficients are larger than 0.95 in the whole sampling time, the similarity is good enough, the whole situation advantage is steady and the game situation is stable, so we can say that the situation advantage in the period do not change, we use Game Theory method and give the best air combat strategy.

\section{Conclusion}

The paper solve UAVs countermeasure selection problem under the complex air combat situation, it proposed to use Time Series Similarity Method to quantitative compare the combat situation information of adjacent time and divide the game situation into "change" or "stable" situation. this paper analyzes the variability of real-time combat situation and continuous sampling the enemy situation information over a period of time, and use the advantage of the situation function to calculate the advantage of our situation and lists the advantages of the overall trend information sequence, then proposed a Time Series similarity method of UAVs combat situation advantage, and make the curve of the situation advantage and the Simulation show that the method feasibility and effectiveness. It is a scientific method that can solve situation advantage similarity problem under the battlefield environment, and it also can provide a reliable technical support for further air combat decision research under the uncertain environment.

\section{Acknowledgements}

I would like to express my gratitude to all those who helped me during the writing of this thesis. My deepest gratitude goes first and foremost to Professor Chen xia, my supervisor, for her constant encouragement and guidance. She has walked me through all the stages of the writing of this thesis. She devotes a considerable portion of her time to reading my manuscripts and making suggestions for further revisions. Her tremendous assistance in developing the framework for analysis and in having gone through the draft versions of this thesis several times as well as her great care in life deserve more thanks than I can find words to express. Without his consistent and illuminating instruction, this thesis could not have reached its present form.

Second, I would like to express my heartfelt gratitude to Professor Xu guang yan, who led me into the world of translation. I am also greatly indebted to the professors and teachers at the Department of English: Ms. Wang Li, Tian wen hua, who have instructed and helped me a lot in the past years.

Last my thanks would go to my beloved family for their loving considerations and great confidence in me all through these years. I also owe my sincere gratitude to my my friends and my fellow classmates who gave me their help and time in listening to me and helping me work out my problems during the difficult course of the thesis.

\section{References}

[1] D. F. Li, "Differential Games and Applications", Defense Industry Press, vol. 4-7, (2000).

[2] W. Getz and G. Leitmann, "Qualitative Differential Games with Two Targets", J Math Anal Apples, vol. 68, no. 3, (1979), pp. 421-430.

[3] G. Leitmann, "Guaranteed Avoidance Strategies", Journal of Optimization Theory and Applications, vol. 32, no.4, (1980), pp. 569-576.

[4] J. X. Li and M. A. Tong, "Multi-objective differential game theory and its application in multi-target", Systems Engineering Theory and Practice, vol. 2, (1996), pp. 28-33.

[5] Y. N. Wang and Y. X. Jiang, "Intelligent Differential Game in air combat measures", Flight Mechanics, vol. 21, no. 1, (2003), pp. 66-70.

[6] H. H. Chin, "Knowledge - Based System of Super maneuver Select ion for Pilot Aiding", Aircraft, vol. 26, no. 12, (1989), pp. 56-66. 
[7] F. X. Zhang, P. H. Wang and F. Li, "Application of Artificial Neural Network to air combat countermeasure system", Electro-optical and Control, vol. 1, (2001), pp. 32-351.

[8] X. Chen, M. Liu and Y. X. Hu, "UAVs attack and defense strategy game based on uncertain information research", Ordnance Journal, vol. 33, no. 12, (2012), pp. 1510-1515.

[9] M. L. Li, "Time-Series Similarity clustering algorithm studies", (2010).

[10] Y. Liu, "The new Time-Series similarity measure studies", (2007).

[11] X. L. Dong, "Time series data mining similarity measure and periodic pattern mining research", (2007).

[12] J. L. Lan and S. H. Zhao, "Cooperative Multiple Target Attack Combat Tactical Decision", Electro-optical and Controlling, vol. 17, no. 12, (2010), pp. 17-19.

[13] P. Pu, C. Jin and J. Xi, "Study on Multi-aircraft cooperative multi-target assignment Tactical Decision", Tactical Missile Technology, (2007), vol. 6, no. 2, pp. 57-61.

[14] Y. Gao and J. W. Xiang, "BVR Air combat target assignment algorithm", Journal of Beijing University of Aeronautics and Astronautics, (2007), vol. 33, no. 3, pp. 286-289.

[15] A. Gal, "Game theoretic target assignment strategies in competitive multi-team systems", University of Pittsburgh, (2004), pp. 1-9.

[16] W. Zhou, Y. Z. Wang and Q. Z. Ma. "Grab the advantage of the game by using the situation changes. Journal of System Simulation”, (2008), vol. 20, no. 17.

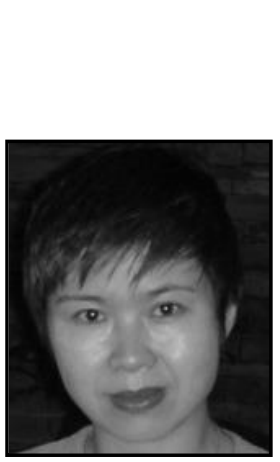

\section{Authors}

Xia Chen, she was born in 1962, she is a professor in Shenyang Aerospace University. Her Main research interests are measurement technique, automatic control, flight control, etc.

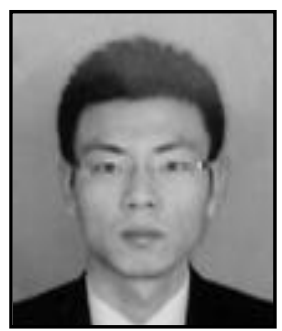

Yifei Wang, he was born in 1988, he is a graduate student in Shenyang Aerospace University .His main research interest is the research of unmanned aerial vehicle (UAV) game theory. 